\title{
CERN Intersecting Storage Rings
}

A general article on the purpose of the ISR and on the major technical features of the project. Although most of this information has been covered in CERN COURIER before, particularly in July 1966, it is repeated here to refresh memories and for the benefit of the many new readers.

\section{K. Johnsen}

The research at CERN is part of what might be considered as the third generation of the scientific attack on the nature of matter. It follows on from the understanding of the nature of the atom and from the understanding (still only partial) of the nucleus at the heart of the atom. Now most effort is being concentrated on the understanding of the individual particles which go, for example, to make the nucleus.

The research has moved progressively to the examination of matter on a smaller and smaller scale - from the atom with dimensions of $10^{-8} \mathrm{~cm}$ (a hundredth of a millionth of a centimetre), to the nucleus of $10^{-12} \mathrm{~cm}$ (ten thousand times smaller) to the particle of about $10^{-13}$ (ten times smaller still). To look at phenomena of these progressively smaller dimensions has, paradoxically, called for larger and larger resources; in particular larger and larger accelerators.

To break into an atom requires very little energy (atoms are 'smashed' for example every time a match is struck). To break into the nucleus needs an energy many millions of times greater, and to break into a particle, thousands of times greater still. These energies are usually expressed in terms of the electronvolt $(\mathrm{eV})$ - the energy given to the electron which is accelerated through a potential difference of 1 volt. To smash atoms requires several tens of $\mathrm{eV}$, nuclei many millions of $\mathrm{eV}(\mathrm{MeV})$ and particles thousands of millions of $\mathrm{eV}(\mathrm{GeV})$.

As higher energies have become available with the development of the technology to build higher and higher energy accelerators, new worlds of phenomena have been revealed. The research using the accelerators has revolutionized the conception of matter, uncovering a large number of previously unknown particle states, revealing the existence of new forces controlling the behaviour of the particles, and overthrowing some intuitive ideas about such things as symmetry in Nature.

The stage has now been reached where many of the questions posed by the current research call for still higher energies to attempt to answer them. It is for this reason that the construction of more powerful machines is beginning or is planned. The present major proton synchrotrons - 28 $\mathrm{GeV}$ at CERN, $33 \mathrm{GeV}$ at Brookhaven (USA) and the recently completed $75 \mathrm{GeV}$ at Serpukhov (USSR) - will be surpassed by the $200-400 \mathrm{GeV}$ machine at Batavia (USA) and the proposed $300 \mathrm{GeV}$ machine in Europe.

\section{Colliding beams}

It has been realized for many years that it would be possible to obtain a glimpse into a much higher energy region if particle beams could be persuaded to collide head-on.

To explain why this is so, we need to consider what happens in a conventional accelerator experiment. When particles have the required energy they are directed onto a target and collide with the stationary particles of the target. Most of the energy given to the accelerated particles then goes into keeping the particles which result from the collision moving in the direction of the incident particles (to conserve momentum). Only a quite modest fraction is 'useful energy' for the real purpose of the experiment - the transformation of particles, the creation of new particles. For example, at the full energy of the CERN $28 \mathrm{GeV}$ synchrotron about 7 $\mathrm{GeV}$ is useful energy. (An approximate formula for determining the useful energy is :

Useful energy $=V$ Twice accelerator energy where the energies are expressed in $\mathrm{GeV}$. Thus a $300 \mathrm{GeV}$ machine gives about 24 $\mathrm{GeV}$ of useful energy.)

But if particles of the same energy were made to collide head-on, all their energy would be useful since none would be needed to conserve momentum, to keep things moving in a particular direction. The fascination of the CERN intersecting storage rings then lies in the prospect of colliding $28 \mathrm{GeV}$ protons head-on and having 56 $\mathrm{GeV}$ of useful energy available. To achieve this with a conventional accelerator would require a machine with an energy of about $1700 \mathrm{GeV}$ which is possibly beyond existing technology and certainly beyond existing financial resources.

This great leap forward in useful energy by using colliding beams has to be qualified by repeating that they will provide a glimpse into a much higher energy region rather than a broad look. The conventional accelerator is a prolific source of many types of particle and it can be used to investigate interactions involving protons, antiprotons, kaons, pions, neutrinos... With colliding beams the interaction is limited to that of the beam particles - for the CERN ISR this means the proton-proton interaction.

\section{The Intersecting Storage Rings}

In December 1965, the CERN Council approved the construction of intersecting storage rings; France had already made available a piece of land across the border from Switzerland to build the ISR. What follows is a general description of the technical features of the machine. (More detailed descriptions of the component parts begin on page 267.)

The ISR consist of two concentric rings of magnets, $300 \mathrm{~m}$ in diameter, in which protons travel in opposite directions. The rings are built in a circular underground tunnel some $200 \mathrm{~m}$ away from the $28 \mathrm{GeV}$ proton synchrotron. The two rings are not exactly circular but are interlaced so that they intersect at eight points, called interaction regions, where the beams can be brought into collision. A schematic representation of the configuration and of the beam paths can be seen in the Figure and the main parameters of the rings are given in the Table.

Protons are accelerated to the required energy (which can be between 8 and 28 $\mathrm{GeV}$ ) in the PS. They are then ejected by a fast-ejection system into a transfer channel in which a magnet system guides them towards the ISR. This channel forks into two and, depending on whether a bending magnet at the fork is switched on or not, the protons go left or right to enter one or the other of the rings. The left and right channels then have to climb up-hill because the beam level in the ISR is about $12 \mathrm{~m}$ higher than in the PS. The protons are injected into a ring by a fast-injection system, so that they initially travel close to the inside wall of the ring vacuum chamber.

If simply one pulse was taken from the PS, containing say $10^{12}$ protons, and fed into one ring and another similar pulse was fed into the other ring orbiting in the opposite direction, the number of collisions per second which would take place 


\section{Main Parameters of the ISR}

Number of rings.

Circumference of rings

Number of intersections

Length of long straight section .

Intersection angle at crossing points .

Maximum energy of each beam

Magnet (one ring)

Maximum field at equilibrium orbit Maximum current to magnet coils.

Maximum power dissipation .

Number of magnet periods.

Number of superperiods

Total weight of steel.

Total weight of copper

2

$942.66 \mathrm{~m}$

8

$16.8 \mathrm{~m}$

$14.7885^{\circ}$

$28 \mathrm{GeV}$

$12 \mathrm{kG}$
$3750 \mathrm{~A}$
$7.04 \mathrm{MW}$
48
4
5000 ton
560 ton

\section{R.F. system (one ring)}

Number of r.f. cavities

Harmonic number

Centre frequency of r.f.

Maximum peak r.f. voltage per turn

\section{Vacuum System}

Vacuum chamber material

Vacuum chamber inside dimensions.

Design pressure outside intersection regions.

Design pressure inside intersection regions.
6

30

$3.53 \mathrm{MHz}$

$20 \mathrm{kV}$

low carbon stainless steel

$160 \times 52 \mathrm{~mm}^{2}$

$10^{-9}$ torr

$10^{-10}$ to $10^{-11}$ torr when the beams met in the interaction regions would be unacceptably small. Experimenters using the beams produced by the PS are used to a hundred thousand collisions per second taking place, say, in a hydrogen target. The ISR has been designed to achieve a similar figure when the beams collide.

To do this, it is necessary to increase the intensity of the two orbiting beams so that they each contain $4 \times 10^{14}$ protons, which is equivalent to a circulating current in each ring of about $20 \mathrm{~A}$. It is achieved by stacking many successive pulses from the PS next to one another.

For this purpose a radio frequency system is needed. After the first pulse has been injected, this r.f. system accelerates the protons just enough to move the particles from their injection orbit to an orbit nearer the outside of the vacuum chamber. When this acceleration has been done the injection orbit is free to receive the next pulse, which, in its turn, is accelerated and moved to an orbit only a fraction of a millimetre from where the first pulse was left. This stacking process can be repeated again and again, in fact about 400 times in each ring, creating a stacked beam about $70 \mathrm{~mm}$ wide with the intensities mentioned above. (Variants of the stacking, procedure are covered later.) With 400 pulses stacked there will be a momentum spread of $2 \%$ across the beam.

The time taken to stack each ring to such an intensity, with the present performance figures of the PS, would be less than an hour and when the improvements programme at the synchrotron is complete; this may fall to as low as five minutes. The protons can circulate in the rings and colliding beam experiments can be carried out for as long as a day before calling on the PS again for a refill.

\section{Special requirements}

Most of the major problems in constructing the ISR arise because of the need to build up intense proton beams and to keep them orbiting in their rings for many hours. The conditions which must be established are very different from the conventional accelerator where the beam is in and out of the machine in the order of a second.

The r.f. system is not required to produce unusually high accelerating voltages but has to be capable of carefully controlled voltage variations from $20 \mathrm{kV}$ at injection to tens of volts at the end of the stacking process. The magnet system has to provide a very precise field configuration to guide and focus the beams and has to incorporate a full range of correction possibilities to cope with any deviations from the ideal in the beam paths. The main magnets have also to provide 'good field' across the full vacuum vessel aperture (over $150 \mathrm{~mm}$ horizontally) up to a field strength of $12 \mathrm{kG}$ on the equilibrium orbit. The lay out of the two magnet rings where they pass between interaction regions 3 and 4 can be seen in Figure 2 .

The demands placed on the vacuum system are particularly: severe. If beams are to be retained in the rings for many millions of turns without serious loss in intensity, not only must the magnet guide fields keep them well under control, but also the number of residual gas molecules that the beams meet must be very small to avoid scattering protons out of the beams. In the conventional accelerator, pressures around $10^{-6}$ torr are adequate ; in the ISR this has to be pushed down to $10^{-9}$ torr (a pressure feasible only in small laboratory-bench set-ups just a few years ago). Taking the two rings together it involves holding a vacuum vessel of a total length of almost $2 \mathrm{~km}$, with thousands of joints, at ultrahigh vacuum. This is by far the biggest ultra-high vacuum system in the world.

Even with this low pressure the scattering caused by the residual gas molecules in the vacuum chamber will make the beams 'blow up' significantly in size over twenty hours and to cater for this, in addition to deviations which could be introduced by imperfections in the magnetic field, the vacuum vessel aperture is set at 160 $\mathrm{mm}$ horizontally and $52 \mathrm{~mm}$ vertically.

Another indication that the stability of the intense beams is fragile is that 'clearing electrodes' have to be installed to sweep away the electrons liberated when the beams ionize the residual gas. These electrons would tend to neutralize the positive charge in the beams and thus upset the delicate balance between the defocusing electric force acting within the beams and the focusing magnetic force that the fast moving charges set up. Without these clearing electrodes only half the planned beam intensity could be stored. Other possible sources of instability have been studied theoretically and some were investigated experimentally using the electron storage ring model CESAR (CERN COURIER vol. 7, page 247) which contributed a great deal to the ISR project, and also using the PS. They are not expected to be troublesome.

\section{Interaction regions}

As mentioned above, the beams can be brought into collision at eight places around the rings. Initially two of these places, numbered 1 and 4 , will be equipped with experimental halls.

When the currents of $20 \mathrm{~A}$ circulating in each ring have been built up, each beam looks like a ribbon $70 \mathrm{~mm}$ wide and $10 \mathrm{~mm}$ high. The ring structure is such that they cross at an angle of $15^{\circ}$ and thus form a volume of about $200 \mathrm{~cm}^{3}$ in which collisions take place. 
A schematic diagram of the relationship between the proton synchrotron and the intersecting storage rings. The beam paths in the PS, the transfer channels and the ISR are shown in blue.

11 and 14 are the experimental halls which are to be built initially

TT1 to TT3 are beam transfer channels

To the West Hall and the $3.7 \mathrm{~m}$ hydrogen bubble chamber
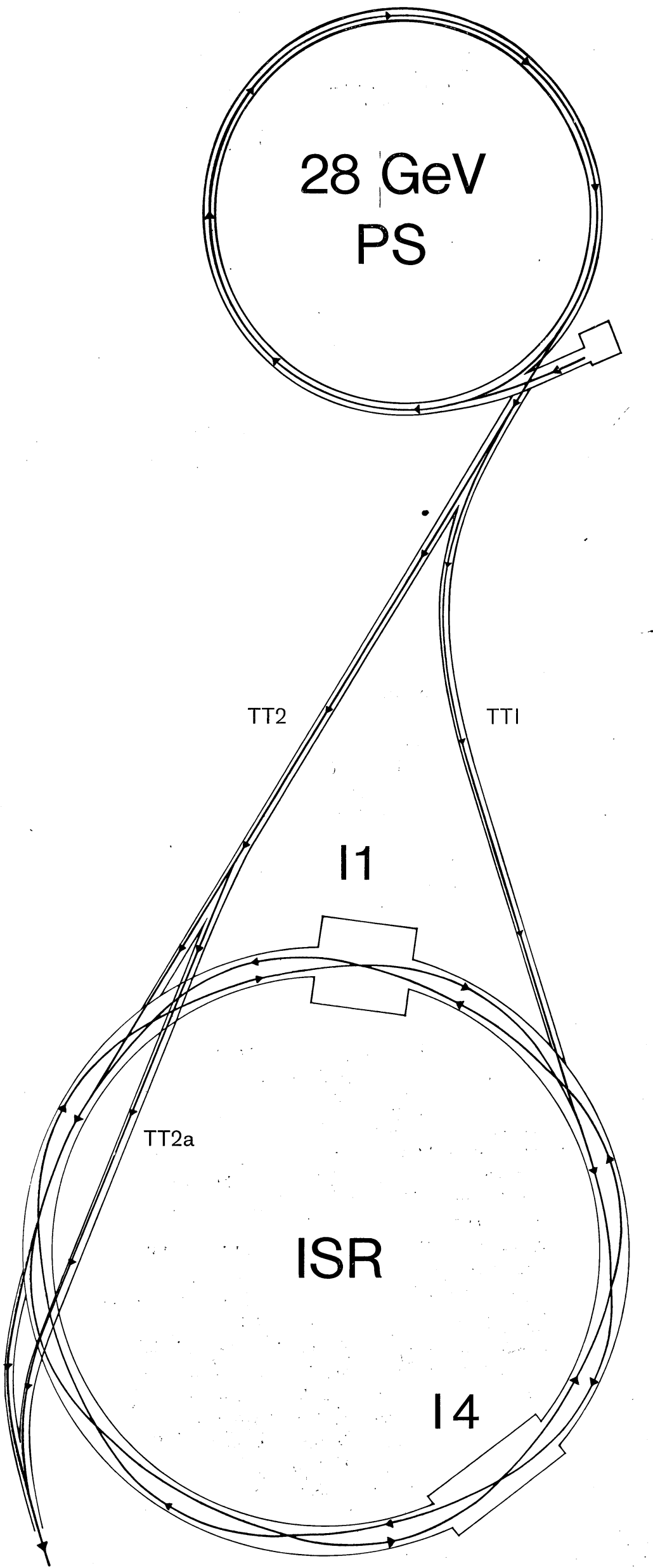
Lay-out of the two magnet rings between interaction regions 13 and 14 . Notice that the outer arc. where most bending has to take place, is packed with magnet, while the inner arc is less congested. It is therefore in the inner arcs that beams are brought in. The two arcs taken in sequence form a 'superperiod', repeated four times around the ring.
The various components are

$F$ Focusing magnet

$D$ Defocusing magnet

$P$ Pick-up station

$\checkmark$ Sector valve

$T$ Terwilliger quadrupole

$Q$ Skew quadrupole

$S$ Sextupole

H Horizontal field magne:

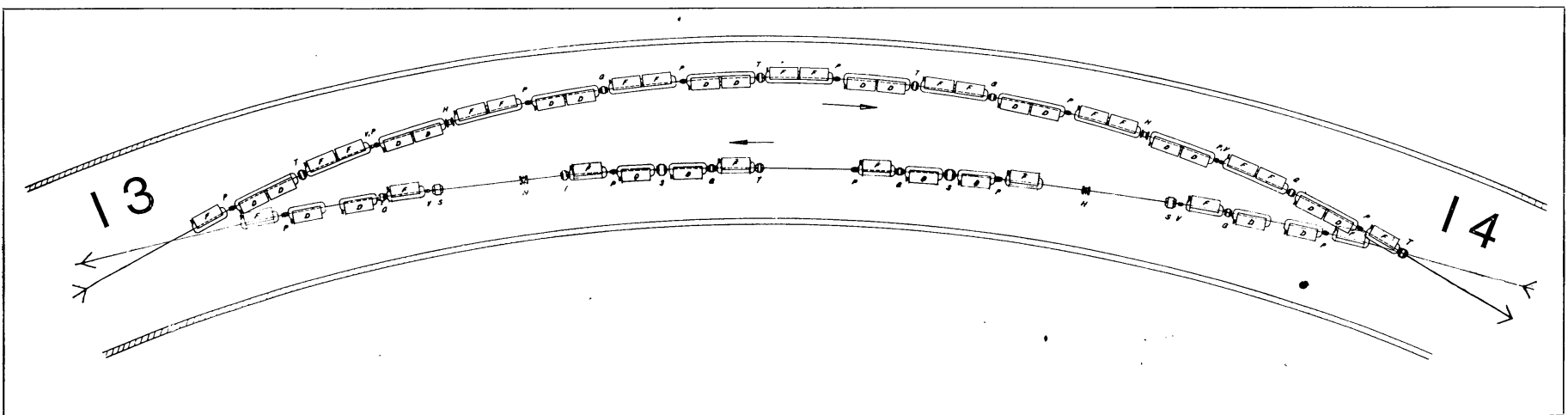

The interaction rate within this volume is proportional to the cross-section of the process one wants to investigate. For this proportionality factor the term 'Iuminosity' is used among colliding beam specialists. The luminosity depends on the geometry of the beams in the interaction region and is proportional to the product of the circulating currents in the two beams. In the ISR, with the beam characteristics as described above, the luminosity is $4 \times 10^{30} \mathrm{per}$ second and per $\mathrm{cm}^{2}$. With a proton-proton total cross-section of $4 \times 10^{-26} \mathrm{~cm}^{2}$ the total interaction rate is $1.6 \times 10^{5} \mathrm{per}$ second. Through its dependence on the stacked beam currents, the luminosity also becomes proportional to the square of the momentum spread of the beams.

When the improvement programme of the proton synchrotron is complete, considerable improvements on these performance figures can be expected. Luminosities may be as much as 70 to 80 times higher than obtainable with the present PS performance, particularly in cases where the experiments have special requirements for low momentum spread that otherwise limit the luminosity to values well below that given above.

During 1967, some new interesting ideas emerged which indicate that it may be possible to increase the performance figures still further, in particular for low momentum-spread experiments. The method consists essentially in not injecting a whole PS pulse (which contains 20 bunches of protons) into the ISR in one go, but in injecting two halves of a pulse (two lots of 10 bunches) separately and with a short and properly chosen interval so that the two halves are superimposed in the injection orbit of the ISR. This is equivalent to a two-turn injection and to a corresponding increase by a factor of two in the local beam intensity. It is then possible to stack this superimposed shorter pulse in such a way that the final stack density goes up by the same factor iwo.

The method can in principle be extended to the equivalent of four-turn injection, but then it will be preferable to do part of the acrobatics between the new PS injector and the synchrotron. The methods proposed require solutions to very difficult technical problems, but there is nevertheless considerably hope that it will be possible, in the future, to take advantage of some of their potentialities and thus gain a further improvement in the luminosity figures.

\section{West Hall and Bubble Chamber}

In conjunction with the ISR proper, further facilities for $28 \mathrm{GeV}$ physics are being built on the same site, particularly a large experimental hall (West Hall) and beyond it the new $3.7 \mathrm{~m}$ hydrogen bubble chamber (see CERN COURIER vol. 7 , page 143). Their relative positions in relation to the ISR can be seen on the site lay-out drawing on page 282.

The hall almost doubles the area fed by beams from the PS which is available for experiments. The beams can reach the hall either via a transfer channel, which branches off from one of the channels supplying the ISR travelling under the ISR and surfacing in a 'switchyard' in front of the hall, or can come from one of the storage rings. The first possibility ensures that experiments can continue in the hall regardless of whether the ISR is in operation or not. The second possibility gives great flexibility in the sort of beam supplied to an experiment - after using the storage capability of the storage ring the experiment could receive an extremely intense beam in one burst or could receive a less intense beam spilled out continuously over a long period of time.

\section{Programme}

The construction programme began at the beginning of 1966. The major excavation work is complete and the tunnel to house the rings is built half way around its circumference.

The large West Hall has been finished as planned and is receiving ISR components, which began to arrive on the site a few months ago, for assembly and testing. By now, magnet components are arriving regularly and several complete units have been put together. About half the radio-frequency cavities are here as well as power supplies for the amplifiers. The deliveries of the sputter ion pumps for the vacuum pumps have also started.

One octant of the ISR tunnel will be handed over for the installation of the magnets etc. by the end of the year. There is every expectation that the project will be completed on schedule in the middle of 1971

Meanwhile the experimentalists are beginning to work out detailed proposals for experiments. The experiments, like the project itself, confront novel problems and will in general need longer to prepare than those at a conventional accelerator. As the day approaches when $56 \mathrm{GeV}$ cf useful energy will be available, the interest in using this unique facility is growing throughout Europe. 